\title{
Comparison of Accuracy Criteria for Approximation of Conservative Fields on Tetrahedra
}

\author{
Igor Tsukerman and Alexander Plaks \\ Electrical Engineering Department, The University of Akron, OH 44325-3904, USA
}

\begin{abstract}
A new maximum eigenvalue criterion for a scaled stiffness matrix and a related singular value criterion for the element 'edge shape matrix', as well as conventional conditions of approximation accuracy, are applied to tetrahedral node and edge elements and compared. The singular value criterion is the most precise and least restrictive $a$ priori condition and is recommended for engineering practice.
\end{abstract}

Index terms - finite elements, accuracy, approximation, interpolation, stiffness matrix, tetrahedral mesh, shape

\section{INTRODUCTION}

Accuracy of Finite Element (FE) approximation is a critical factor in FE analysis. The accuracy depends on the type, size and shape of the elements chosen. Dependence on size $h$ usually has the asymptotic form $O\left(h^{m}\right)$, where $m$ is the order of approximation. Dependence on shape has been studied in detail for first order 2D triangular elements, and the minimum and maximum angle conditions have been proposed [1, 2].

In $3 \mathrm{D}$, first order tetrahedral node elements and edge elements are widely used, but it is not yet well understood what shape related parameters are essential for the approximation accuracy and why. For example, the comparison of various conditions for tetrahedra in [3] is based on a purely geometric reasoning rather than analysis of approximation errors. A common theoretical condition in the mathematical literature is the ratio of the radius of the inscribed sphere to the maximum edge of the tetrahedron $[4, \S 3.1]$.

A new general accuracy criterion, applicable to tetrahedral and other 2D and 3D elements, was proposed in [5]. The maximum eigenvalue of a (scaled) element stiffness matrix was proved to control approximation errors, and the relevant accuracy estimates were obtained.

A more specific study of the maximum eigenvalue criterion for tetrahedral elements is carried out in [6]. It is shown that the maximum eigenvalue of the scaled stiffness matrix is related to the minimum singular value of a properly defined 'edge shape matrix'.

This paper presents numerical experiments comparing various accuracy criteria for tetrahedral meshes. Only criteria with a solid theoretical basis were considered. Central in the comparison is the singular value condition that, due to the theory developed in $[5,6]$, is thought to be the most precise.

Manuscript received November 3, 1997. The work was supported in part by the National Science Foundation research grant ECS-9702364.

I. Tsukerman, itsukerman@uakron.edu, http://www.ecgf.uakron.edu/ igor A. Plaks, aplaks@uakron.edu

\section{THE MAXIMUM EIgENVALUE CRITERION}

In FEM, the exact solution $u_{*}$ of a boundary value problem in a domain $\Omega$ is approximated by a linear combination $\tilde{u}$ of basis functions $\left\{\phi_{i}\right\}(i=1,2, \ldots, n)$ :

$$
\tilde{u}=\sum_{i=1}^{n} u_{i} \phi_{i} \in \tilde{W}
$$

with a vector of degrees of freedom (d.o.f.) $u=\left\{u_{1}, u_{1}, \ldots\right.$, $\left.u_{n}\right)^{T} \in R^{n} . \quad \tilde{W}$ in (1) is the finite dimensional space spanned by the basis functions $\left\{\phi_{i}\right\}$, and the d.o.f. $u_{i}$ in (1) usually represent the node or edge values on a given mesh.

The error of approximating the exact potential $u_{*}$ by $\tilde{u}$ is evaluated in a suitable energy (semi)norm $\|\cdot\|_{E}$ :

$$
\text { approximation error } \varepsilon_{a}=\min _{\tilde{u} \in \tilde{W}}\left\|\tilde{u}-u_{*}\right\|_{E}
$$

For example, in electro- or magnetostatic problems this (semi)norm is usually taken to correspond to the field energy:

$$
\left\|u_{*}\right\|_{E}=\left[\int_{\Omega}\left|\nabla u_{*}\right|^{2} d \Omega\right]^{1 / 2}
$$

It follows from the Lax-Milgram and Céa's lemmas (see e.g. [4]) that the error of the FE-Galerkin solution is equal to the approximation error in (2). For eddy current problems, the solution error generally exceeds the approximation error, but not more than by a constant factor (typically $\sqrt{2}$ [7]). In short, the approximation error (2) is the main governing factor of the solution accuracy.

Important in a theoretical analysis of approximation is the standard interpolating function $\tilde{u}_{I}$ :

$$
\tilde{u}_{I}=\sum_{i=1}^{n} u_{I i} \phi_{i} \in \tilde{W}
$$

where $u_{I i}$ correspond to the d.o.f. for $u_{*}$ (for nodal elements, $u_{I i}$ are the node values of $u_{*}$, etc.). The interpolation error is

$$
\text { interpolation error } \varepsilon_{I}=\left\|\tilde{u}_{I}-u_{*}\right\|_{E}
$$

Clearly, $\varepsilon_{a} \leq \varepsilon_{I}$. Approximation and interpolation errors depend on the chosen set of approximating functions $\left\{\phi_{i}\right\}$, which in the FE context implies the type, size and shape of finite elements. Dependence on shape is reflected in the general accuracy estimate derived in [5] and presented here in a slightly simplified form: 
$\varepsilon_{a} \leq \varepsilon_{I} \leq c\left[\sum_{(e) \in m e s h}\left(\lambda_{\max }\left(\hat{A}^{(e)}\right) h^{(e) 2 \kappa}+h^{(e), 2(m+1-p)}\right) V^{(e)}\right]^{1 / 2}(6)$

The notation in this formula is as follows. The scaled stiffness matrix is $\hat{A}^{(e)}=A^{(e)} / V^{(e)}$, where $A^{(e)}$ is the standard element stiffness matrix corresponding to the chosen energy norm and $V^{(e)}$ is the volume of a finite element $(e) ; \lambda_{\max }$ denotes the maximum eigenvalue of a matrix; $h^{(e)}$ is the element diameter; $c$ is a generic constant independent of the element shape; $m$ is the order of a local Taylor approximation; $\kappa$ is the order of the Taylor approximation of the d.o.f. of the exact solution. As an example, for first order tetrahedral nodal elements $m=1$, and the d.o.f. (the node values) are approximated with $\kappa=2$ (provided the exact potential is sufficiently smooth). Finally, $p$ is the order of the highest derivative included in the energy norm (e.g. $p=1$ for the energy norm (3)).

For approximation of conservative fields on tetrahedral edge elements, it is shown in [6] that the maximum eigenvalue of the scaled element stiffness matrix $\hat{A}^{(e)}$ is directly related to the minimum singular value of the element 'edge shape matrix', as explained in the following section.

\section{The Minimum Singular VAlue CRITERION}

The shape of a tetrahedral element can be characterized by a $3 \times 6$ edge shape matrix $E$ whose columns are the unit vectors $e_{i}(i=1,2, \ldots, 6)$ directed (in either of the two possible directions) along the element edges:

$E=\left[e_{1}\left|e_{2}\right| e_{3}\left|e_{4}\right| e_{5} \mid e_{6}\right]=\left[\begin{array}{llllll}e_{1 x} & e_{2 x} & e_{3 x} & e_{4 x} & e_{5 x} & e_{6 x} \\ e_{1 y} & e_{2 y} & e_{3 y} & e_{4 y} & e_{5 y} & e_{6 y} \\ e_{1 z} & e_{2 z} & e_{3 z} & e_{4 z} & e_{5 z} & e_{6 z}\end{array}\right]$ (7)

The minimum singular value $\sigma_{\text {min }}$ of this matrix characterizes the 'flatness' of a tetrahedral element [6]. This singular value can be zero only for a degenerate tetrahedron whose all six edges are coplanar.

Let a conservative field $H_{*}=\nabla u_{*}$ be approximated by $\tilde{H}=\nabla \tilde{u}$, where $\tilde{u}$ is a piecewise-linear node element potential and $\tilde{H}$ is piecewise-constant. (Note that the Whitney element approximation of the field is completely equivalent to the node element approximation of the potential.) Formally, $\tilde{H} \in \tilde{W}_{\text {const }}^{(e)}$, where $\tilde{W}_{\text {const }}^{(e)}$ is the space of vector fields defined within a particular element $(e)$ and constant over that element. Consider the edge element representation of $\tilde{H}$ :

$$
\tilde{H}=\sum_{1 \leq k \leq 6} H_{k \tau} w_{k}^{\prime},
$$

Here $H_{k \tau}$ are the tangential components of $\tilde{H}$ along the element edges and $w_{k}^{\prime}$ are the scaled edge element basis functions

$$
w_{k}^{\prime}=w_{k} l_{k}, \quad w_{k}=\phi_{i} \nabla \phi_{j}-\phi_{j} \nabla \phi_{i}
$$

where $l_{k}$ are edge lengths, and $w_{k}$ are the regular edge basis functions [8]. In the representation (8), the d.o.f. are the tangential components of the field, their Taylor approximation being of the order

$$
\kappa=1
$$

For the energy norm

$$
\|H\|_{E}=\left(\int_{\Omega}|H|^{2} d \Omega\right)^{1 / 2}
$$

the entries of the regular and scaled element stiffness matrices are, respectively,

$$
A_{i j}^{(e)}=\int_{(e)} w_{i}^{\prime} \cdot w_{j}^{\prime} d V ; \quad \hat{A}_{i j}^{(e)}=\left(1 / V^{(e)}\right) \int_{(e)} w_{i}^{\prime} \cdot w_{j}^{\prime} d V
$$

The theory of [5] and the resultant maximum eigenvalue criterion are interpreted in case of conservative fields as follows. $\hat{A}^{(e)}$ can be viewed as an operator restricted to $\tilde{W}_{\text {const }}^{(e)}$

(that is, applied only to fields $\tilde{H}$ constant within an element). With this restriction, there is a simple link [6] between the maximum eigenvalue of $\hat{A}^{(e)}$ and the minimum singular value of $E$ :

$$
\lambda_{\max }\left(\hat{A}^{(e)}{ }_{\mid \tilde{W}_{\text {const }}^{(e)}}\right)=\sigma_{\min }^{-2}(E)
$$

Then, with $m=0, \kappa=1$ (10) and $p=0$ for (11), the general error estimate (6) becomes

$$
\varepsilon_{a} \leq \varepsilon_{I} \leq c\left[\sum_{(e) \in \text { mesh }}\left(\sigma_{\min }^{-2}\left(E^{(e)}\right)+1\right) h^{(e) 2} V^{(e)}\right]^{1 / 2}
$$

It is easy to verify that $\sigma_{\min }$ is bounded from above ${ }^{1}$. Therefore the first term in the sum (13) is dominant, and ${ }^{2}$

$$
\varepsilon_{a} \leq \varepsilon_{I} \leq c\left[\sum_{(e) \in \text { mesh }} \sigma_{\min }^{-2}\left(E^{(e)}\right) h^{(e) 2} V^{(e)}\right]^{1 / 2}
$$

This estimate can be further simplified. For example,

$$
\varepsilon_{a} \leq \varepsilon_{I} \leq c \sqrt{V} \max _{(e) \in \text { mesh }} \sigma_{\min }^{-1}\left(E^{(e)}\right) h^{(e)}
$$

where $V$ is the volume of the whole domain $\Omega$.

It follows from (14) or (15) that the minimum singular value of the edge shape matrix characterizes the accuracy of FE approximation of conservative fields on tetrahedral elements (i.e. either the node element approximation of the potential or the Whitney element approximation of the field). This singular value condition is less restrictive for the element shape than possible angle criteria [6].

\footnotetext{
1 In fact, $\sigma_{\min }(E) \leq \sqrt{2}$ because $\sigma_{\min }^{2}(E) \leq\left(\sum_{1 \leq i \leq 3} \sigma_{i}^{2}(E)\right) / 3=$ $\left(\sum_{1 \leq i \leq 6} \lambda_{i}\left(E^{T} E\right)\right) / 3=\operatorname{tr}\left(E^{T} E\right) / 3=6 / 3=2$.

2 Note that $c$ is a generic constant that may be different in different expressions.
} 


\section{COMPARISON OF DIFFERENT ACCURACY MEASURES}

\section{A. Choice of Criteria}

Out of the numerous mesh quality criteria available, we have selected the ones that have a solid theoretical basis linking them to the approximation accuracy:

1. [6] The inverse of the minimum singular value, $\sigma_{\min }^{-1}(E)$. This sufficient criterion is the least restrictive of all.

2. [4] The ratio $h^{(e)} / r^{(e)}$ of the maximum edge to the radius of the inscribed sphere.

3. [5] The angle criterion: $1 / \sin \alpha$, where $\alpha$ is the minimum or maximum angle between edges and faces of a tetrahedron. This criterion follows from the maximum eigenvalue condition for the scaled nodal stiffness matrix.

Remark. All of the above criteria characterize only the shape of an element and are independent of its size.

\section{B. Numerical Experiments}

The idea of the first set of experiments is similar to [3]. A regular (equilateral) tetrahedron is transformed by moving its apex (Fig. 1) up and down, so that the tetrahedron changes from flat at the lowest point of the apex to 'needle-like' at the highest point.

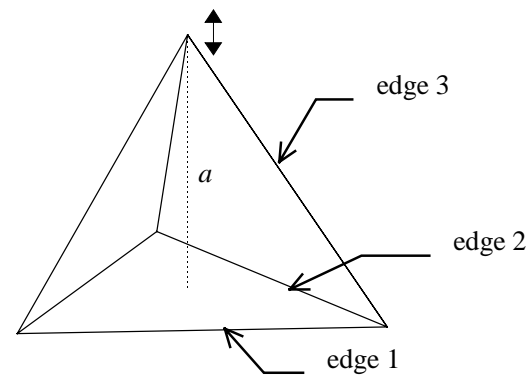

Fig. 1. A tetrahedron with varying height.

Fig. 2 shows the values of different criteria versus height $a$ of the tetrahedron.

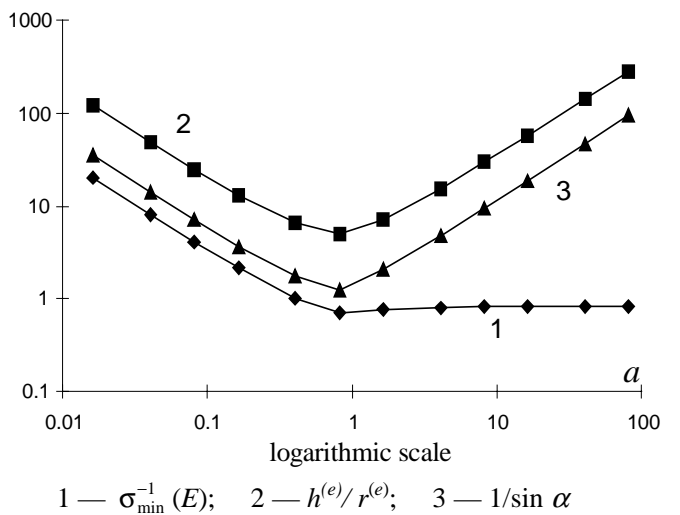

Fig. 2 Values of three criteria versus height $a$ of the tetrahedron.

In the limit $a \rightarrow \infty$, when the apex moves upward, at least three unit edge vectors (e.g. corresponding to edges 1,2,3 in Fig. 1) remain linearly independent, and the minimum singular value of the $E$ matrix remains bounded away from zero. The singular value condition then shows that a 'needlelike' tetrahedron is acceptable, even though it would be rejected on the basis of the minimum angle or the $h / r$ criteria. Flat tetrahedra (with height $a$ tending to zero) are 'truly bad' and are rejected by all criteria.

Fig. 3 shows the results of a similar test for the 'rightangle' tetrahedron, with three edges directed along the coordinate axes and two of them having equal length. Because of the presence of the three orthogonal edges, $\sigma_{\min }(E)$ remains constant and equal to one. Therefore any (even 'needle-like' or 'flat') 'right-angle' tetrahedron is acceptable from the viewpoint of FEM accuracy.

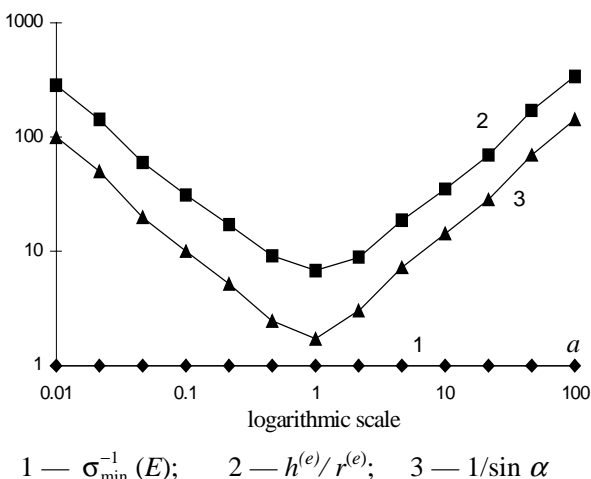

Fig. 3 Values of three criteria for the 'right-angle' tetrahedron vs. height $a$.

The theory in [5, 6] and Figs. 2, 3 suggest that mesh composed of flat or 'needle-like' tetrahedra with three orthogonal edges will give reasonable approximation accuracy despite high aspect ratios and the presence of small angles. The $h / r$ criterion will tend to overestimate the approximation error.

To verify this conclusion, we solved, as a test problem, the Laplace equation in a unit cube. The boundary conditions were consistent with simple analytical solutions similar to $u_{*}=\sin (x) \cos (y) \cosh (\sqrt{2} z)$.

To generate the mesh, the domain was first subdivided into equal rectangular parallelepipeds with dimensions $D_{x}, D_{y}$, $D_{z}$, where $D_{y}=D_{z}=D_{x} / k$, and the aspect ratio $k$ varied from unity to 64 . Then each parallelepiped was split into six tetrahedra. For large values of $k$, this mesh contains 'needle-like' elements. Fig. 4 shows the actual relative error of FE computations

$$
\varepsilon_{\text {actual }}=\left[\sum_{(e) \in \text { mesh }}\left|\nabla \tilde{u}-\nabla u_{*}\right|^{2} V^{(e)} / \sum_{(e) \in \text { mesh }}\left|\nabla u_{*}\right|^{2} V^{(e)}\right]^{1 / 2}
$$

versus the singular value error estimate

$$
\varepsilon_{\sigma}=\left[\sum_{(e) \in \text { mesh }}\left(\sigma_{\min }^{-1}\left(E^{(e)}\right) h^{(e)}\right)^{2} V^{(e)}\right]^{1 / 2}(\text { see }(14)) .
$$

The overall linear correlation between $\varepsilon_{\text {actual }}$ and $\varepsilon_{\sigma}$ is evident from Fig. 4, which means that the global accuracy of FEM is indeed consistent with the a priori singular value estimate. 


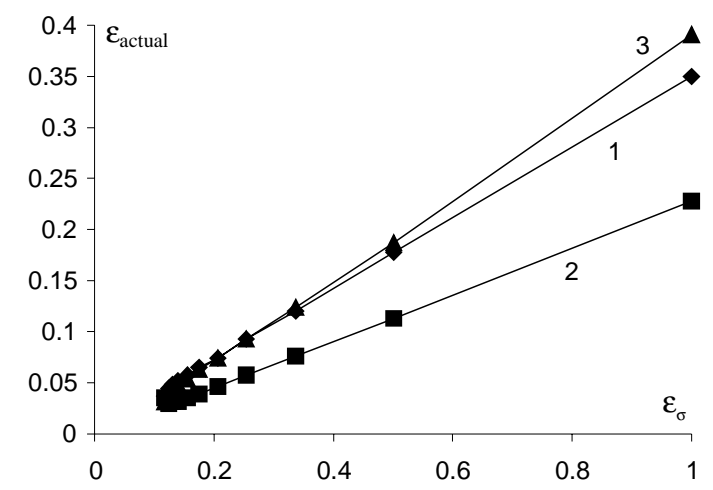

$1-u_{*}=\sin (x) \cos (y) \cosh (\sqrt{2} z) ; \quad 2-u_{*}=\sin (z) \cos (x) \cosh (\sqrt{2} y)$

$$
3-u_{*}=\sin (y) \cos (z) \cosh (\sqrt{2} x)
$$

Fig. 4. Global relative error vs. the singular value estimate for elements with aspect ratio $k$ varying from one to 64 .

Fig. 5 shows $\varepsilon_{\text {actual }}$ versus the $h / r$ estimate [4]

$$
\varepsilon_{h / r}=\left[\sum_{(e) \in \text { mesh }}\left(h^{(e) 2} / r^{(e)}\right)^{2} V^{(e)}\right]^{1 / 2}
$$

The $h / r$ criterion tends to overestimate the error for 'needlelike' elements and therefore the plot lines in Fig. 5 are curved.

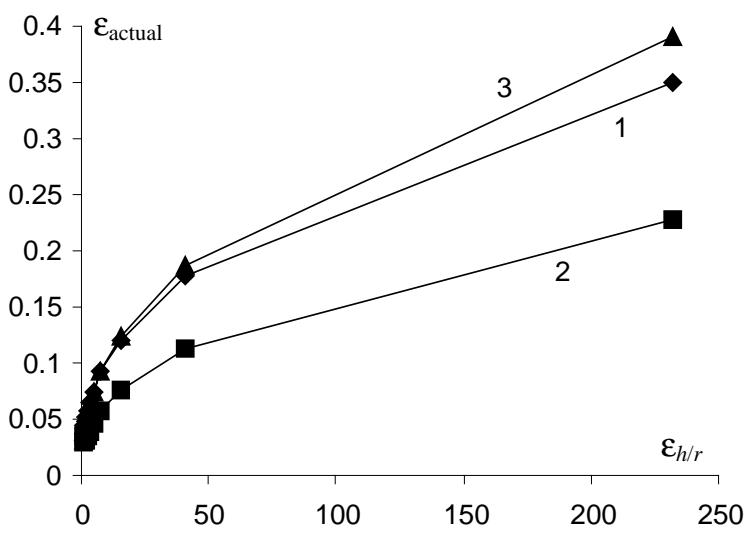

Fig. 5. Global relative error versus the $h / r$ estimate for elements with aspect ratio $k$ varying from one to 64 . Test solutions $1,2,3$ are the same as in Fig. 4.

The difference between two estimates is significant for 'needle-like' tetrahedra but less evident for elements with 'reasonable' aspect ratios. This is illustrated in Fig. 6, 7. The problem with the exact solution $u_{*}=\sin (x) \cos (y) \cosh (\sqrt{2} z)$ was solved on 18 different meshes with $0.25 \leq D_{x} / D_{y}, D_{y} / D_{z}$, $D_{z} / D_{x} \leq 4$. For such moderate aspect ratios, each of the $a$ priori estimates is linearly correlated with the actual error.

\section{CONCLUSIONS}

1. The $h / r$ and angle criteria tend to overestimate the interpolation error for 'needle-like' tetrahedral elements, which may make these estimates too restrictive.
2. Overall, the singular value estimate gives the most consistent predictions and appears to be the most precise $a$ priori condition for the shape of tetrahedral elements.

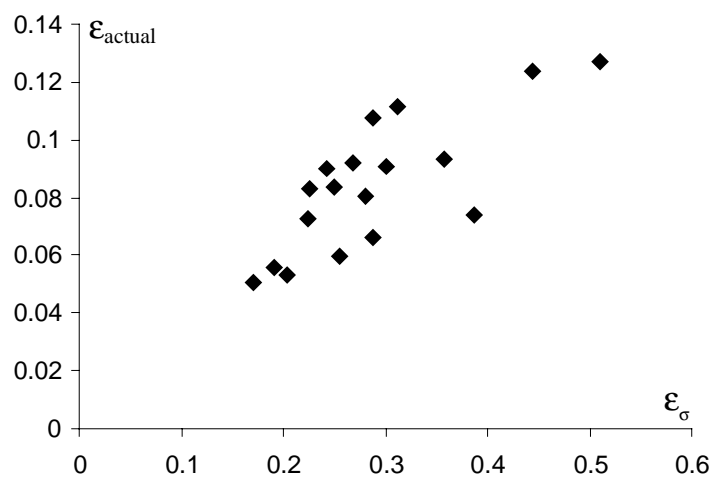

Fig. 6. Actual error versus the singular value estimate for elements of 'reasonable' shape (aspect ratio $k$ from 0.25 to 4 ). Results for 18 meshes.

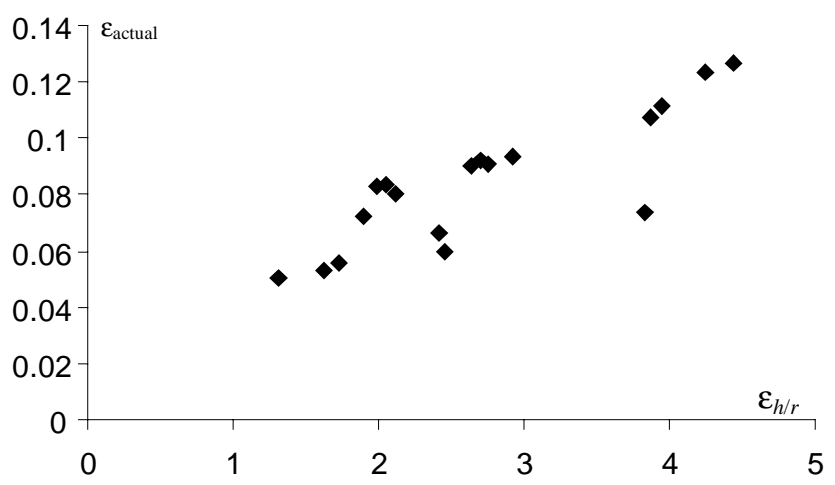

Fig. 7. Actual error versus the $h / r$ criterion for elements of 'reasonable' shape (aspect ratio $k$ from 0.25 to 4 ). Results for 18 meshes.

\section{ACKNOWLEDGMENT}

Igor Tsukerman is grateful to Drs. Alain Bossavit and Pierre Asselin for a very useful discussion of FE accuracy vis-àvis the element shape.

\section{REFERENCES}

[1] M. Zlámal, "On the finite element method," Numer. Math., vol. 12, pp.394-409, 1968.

[2] I. Babuška, A.K. Aziz, "On the angle condition in the finite element method, SIAM J. Numer. Anal., vol. 13, No. 2, pp.214-226, 1976.

[3] V.N. Parthasarathy, C.M. Graichen, and A.F. Hathaway, "A comparison of tetrahedron quality measures," Finite Elements in Analysis and Design, vol. 15, 1993, pp.255-261.

[4] P.G. Ciarlet, The Finite Element Method for Elliptic Problems, NorthHolland: Amsterdam, 1978.

[5] I. Tsukerman, "A general accuracy criterion for finite element approximation," this journal.

[6] I. Tsukerman, "Approximation of conservative fields and the element 'shape matrix'," this journal.

[7] I.A. Tsukerman, "Error estimation for finite element solutions of the eddy currents problem," COMPEL, vol. 9, No. 2, pp.83-98, 1990.

[8] A. Bossavit, "A rationale for "edge-elements" in 3-D fields computations," IEEE Trans. Magn., vol.24, No. 1, pp.74-79, 1988. 